\title{
Advanced Distillation Curve Measurement with a Model Predictive Temperature Controller
}

\author{
B. L. Smith ${ }^{1}$ and T. J. Bruno ${ }^{1,2}$
}

\author{
Received March 7, 2006
}

\begin{abstract}
In previous work, several significant improvements in the measurement of distillation curves for complex fluids were introduced. The modifications to the classical measurement provide for (1) temperature and volume measurement(s) of low uncertainty, and most important, (2) a composition-explicit data channel in addition to the usual temperature-volume relationship. This latter modification is achieved with a new sampling approach that allows precise qualitative as well as quantitative analyses of each fraction, on the fly. In the new approach, the distillation temperature is measured in two locations. The temperature is measured in the usual location, at the bottom of the take-off in the distillation head, but it is also measured directly in the fluid. We have further modified our developmental instrument to incorporate a model predictive temperature controller. In response to either an equation-of-state calculation or a previous distillation curve, the programmable temperature controller increases the fluid temperature to achieve a constant mass flow rate of vapor through the distillation head. This approach eliminates the aberrations that one typically encounters in the data due to fluctuations in distillation rate, often referred to as hesitation. Thus, we can collect data from two temperature channels: one a true state point measurement (measured directly in the fluid) and the other comparable to previous data (measured in the head).
\end{abstract}

KEY WORDS: boiling curve; complex fluids; distillation curve; hesitation; hydrocarbons; model predictive temperature controller.

\section{INTRODUCTION}

One of the most important and informative properties that is measured for complex fluid mixtures is the distillation (or boiling) curve [1]. Simply

\footnotetext{
${ }^{1}$ Physical and Chemical Properties Division, National Institute of Standards and Technology, 325 Broadway, Boulder, CO 80305-3328, U.S.A.

${ }^{2}$ To whom correspondence should be addressed. E-mail: bruno@boulder.nist.gov
} 
stated, the distillation curve is a graphical depiction of the boiling temperature of a fluid mixture plotted against the volume fraction distilled. Alternatively, the data can be presented in (limited) tabular form in fluid specifications and on material safety data sheets (MSDS). The simplest apparatus for the measurement of distillation curves as set forth in the ASTM D-86 standard embodies a Bunsen burner chamber for heating, a liquid bath with a condensate tube, and a graduated cylinder covered with blotting paper for a calibrated volume receiver [2]. This most basic approach suffers from numerous disadvantages. There are clear hazards associated with an open flame, even if it is shrouded in a heating chamber, when applied to hydrocarbon fluids. The use of a graduated cylinder as a receiver is also problematic because distillate tends to splash into the cylinder, making a precise volume measurement as a function of time difficult if not impossible. Deflectors are sometimes used to ameliorate (but not eliminate) this difficulty. This basic approach is embodied in several commercial instruments that function under essentially manual control.

The general importance of the distillation curve has led to the availability of other commercial instruments that employ electrical resistance heating, and automated level-following (an optical method for meniscus detection) for the volume measurement and to control distillation rate [3]. While these commercial instruments provide improvement in terms of convenience, there have been both serious shortcomings and opportunities for improvement. The commercial devices determine the initial boiling temperature (IBT) with a photocell that detects the first drop to fall into the receiver. This clearly produces a delayed response that usually overstates the IBT that is reported by these instruments. This delay results in a significant systematic uncertainty in the IBT; it is impossible to regard this value as a state point. Moreover, it is not uncommon for complex fluids to show an initial vaporization (noted by the onset of bubbles) followed by a lull, then the onset of sustained boiling. This subtle behavior is missed when using the commercial devices. In addition, one typically selects from a menu of programmed heating profiles for a given fluid (such as a gasoline), and this pre-selected heating profile will influence the measured value of the IBT. Also, since the distillation flask is not stirred in the commercial devices, the potential for local superheating and nonuniformity in the horizontal direction is significant.

While the shortcomings discussed above are very serious, the information obtained from such instruments can be useful nonetheless for coarse quality control and oversight applications, especially given that most operators follow the same procedures. The current approaches cannot be used for most diagnostic or detailed applications, however. Moreover, far greater demands are placed on measurements by the desire to 
model fluid behavior with, for example, equations of state, and for this, the current approach is all but useless. Such equation-of-state models are required, for example, in the development of advanced technologies and in the optimization of existing technologies, where full system simulations may be desirable. In these circumstances, there is a need for measurements that are understood at a fundamental level, with much lower uncertainties, and with none of the instrument dependences found with commercial devices. Additionally, the information content of the distillation curve can be greatly expanded by adding a composition channel to the temperature channel. By this is meant an explicit composition measurement (both qualitative and quantitative) for each boiling fraction. This is important for even complex fluids because the actual information that is desired from a distillation is some understanding of how the composition varies with volume fraction and boiling temperature. This is the case whether the distillation is done to design or affect a separation (fractional distillation), or as a material characterization test (simple distillation).

To address the shortcomings discussed above, improvements have been introduced that are depicted schematically in Fig. 1, with additional details provided in Figs. 2 and 3 [4-6]. Since the major modifications have been discussed in detail elsewhere, only a summary will be provided here. The distillation flask is a $500-\mathrm{mL}$ round bottom flask that is placed in a two-part aluminum-heating jacket (Alloy 6061), the lower part of which is contoured to fit the flask. The upper part is placed around the flask after the flask has been inserted into the contoured (lower) part of the

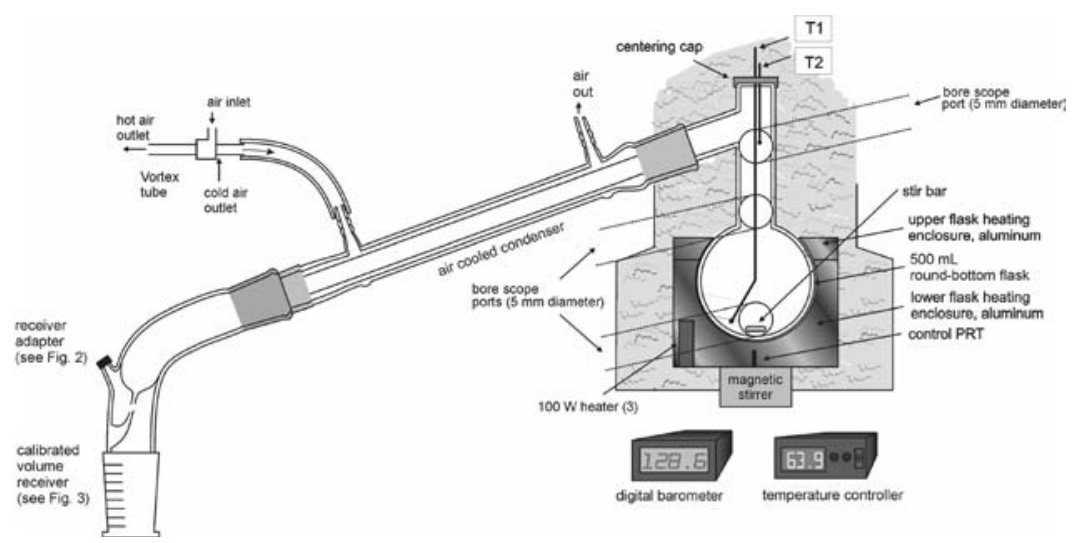

Fig. 1. Schematic diagram of the overall apparatus used for the measurement of distillation curves. Note that the size of the borescope observation ports are only $5 \mathrm{~mm}$ in diameter. Size is exaggerated on the figure to make the location clear. 


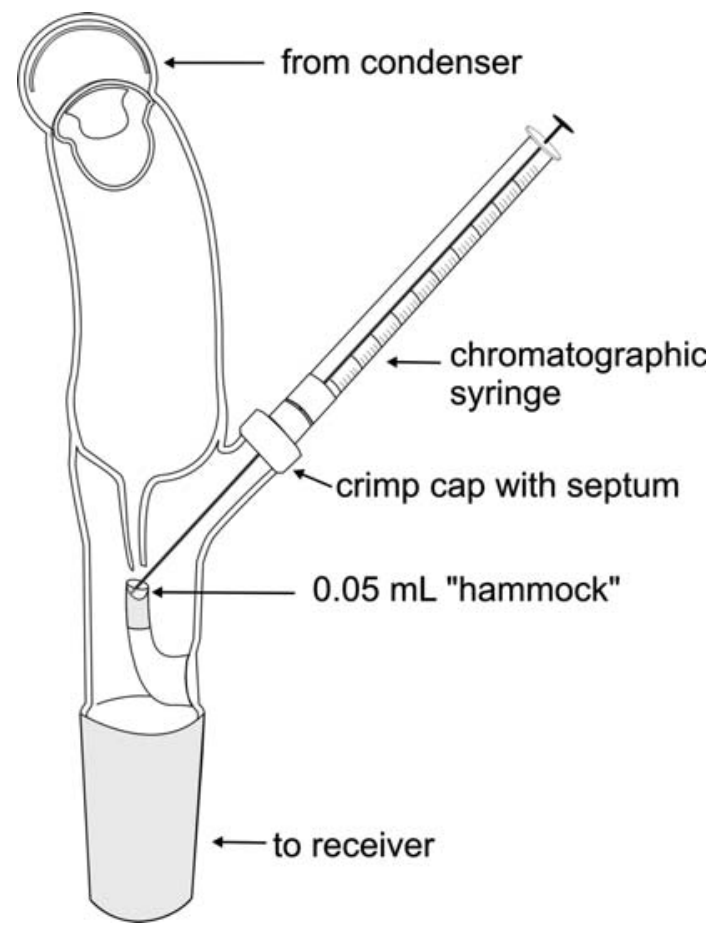

Fig. 2. Schematic diagram of the receiver adapter developed for this work to provide on-the-fly sampling of distillate cuts for subsequent chromatographic analysis.

jacket. This two-part enclosure effectively surrounds approximately $4 / 5$ of the spherical section of the flask. Three cartridge heaters are placed in the lower, contoured part of the jacket, arranged in a "Y" pattern, to provide uniform heating axially about the radius of the enclosure. Heating the flask in this way produces a temperature gradient in the vertical direction, while providing temperature uniformity in the horizontal direction.

The jacket and heaters are capable of operation up to $350^{\circ} \mathrm{C}$, with a local uniformity of $0.2^{\circ} \mathrm{C}$. Note that this is the combined uncertainty in the control as well as the uniformity. The jacket exterior is insulated with a Pyrex wool enclosure. This insulation extends the entire length of the distillation head. This ensures minimal heat leak in the vertical direction, and therefore a small, constant temperature gradient to minimize refluxing. Three observation ports are provided in the insulation to allow penetration with a flexible, illuminated bore scope. The bore scope ports, illustrated in Fig. 1, are placed to observe the fluid in the boiling flask, the top of the boiling flask (where the spherical section joins the head, and the 


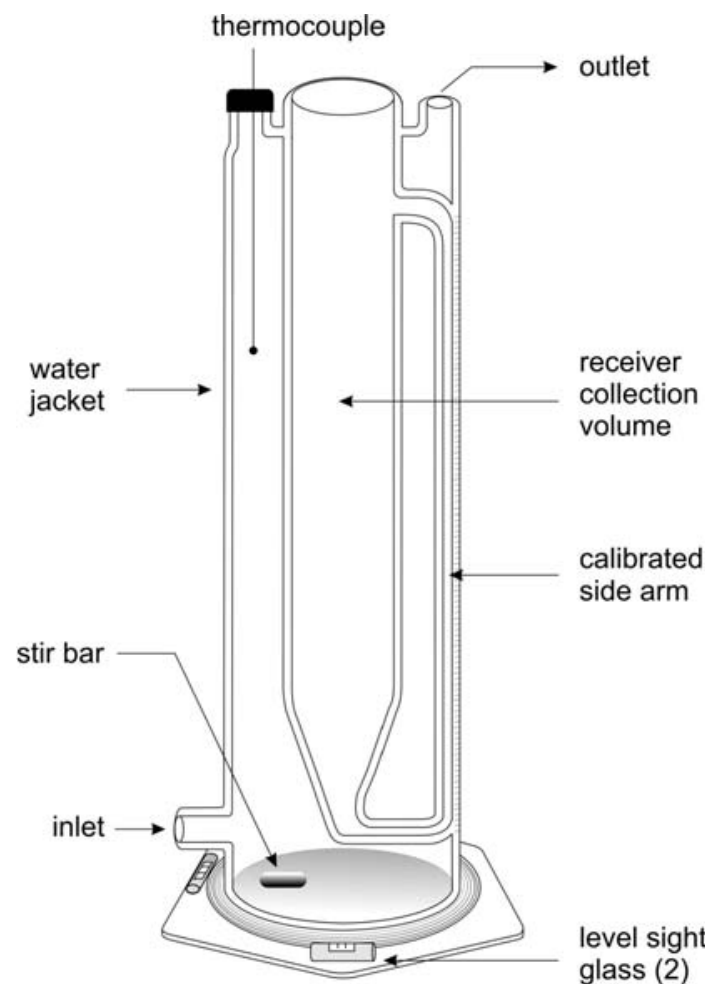

Fig. 3. Schematic diagram of the level-stabilized receiver developed for this work.

distillation head (at the bottom of the take-off). Note that the size of each of the bore scope ports is only $5 \mathrm{~mm}$ in diameter; the size is exaggerated in the figure simply to make the port locations clear. The use of bore scope ports for observation prevents unnecessary heat loss and subsequent loss of temperature uniformity in the horizontal direction.

Above the distillation flask, a centering adapter provides access for two thermally tempered J-type thermocouples that enter the distillation head. One thermocouple (T1 in Fig. 1) enters the distillation flask and is submerged in the fluid, to monitor the temperature of the bulk fluid. This temperature is referred to as $T_{\mathrm{k}}$ (signifying its placement in the kettle). The other thermocouple (T2 in Fig. 1) is centered at the low point of distillate take-off (the typical distillation head placement, as recommended graphically in ASTM-86). This temperature is referred to as $T_{\mathrm{h}}$ (signifying its placement in the head). Both of these thermocouples were calibrated in an indium triple point cell traceable to a NIST standard. 
Monitoring the bulk fluid temperature in addition to the head temperature is valuable for modeling studies, and provides lower uncertainty in the overall test method. This thermocouple is located well below the surface of the fluid. The thermocouples positioned as stated provide a rapid response to temperature, and have a specified uncertainty (from the manufacturer) of $0.05^{\circ} \mathrm{C}$.

Beneath the aluminum jacket, a magnetic stirrer drive is positioned to couple with a magnetic stir bar inside the distillation flask. Rapidly stirring the contents of the distillation flask during the measurement is essential for maintaining horizontal temperature uniformity in the fluid. Indeed, although the cartridge heaters can provide a uniformity of $0.2^{\circ} \mathrm{C}$, stirring integrates variations throughout the volume, allowing the measured temperature from $\mathrm{T} 1$ to be much more uniform and less uncertain. Since a vortex forms on the surface of the fluid because of the stirring, it is necessary to decrease the stirring rate as the measurement progresses, in order to prevent premature lift-out of the thermocouple, T1, from the fluid. The lift-out of T1 from the fluid is an inevitable result of the end of the measurement, however, and is a very useful indication of the end of a measurement. This is easily recognized as a sudden upturn in temperature, and will be illustrated below.

Distillate is taken off the flask with a distillation head, into a forced-air cooled condenser. Following the condenser, the distillate enters a newly designed transfer adapter that allows instantaneous sampling of distillate for chromatographic analysis. The position of the transfer adapter is shown in Fig. 1, and is illustrated schematically in more detail in Fig. 2 [5]. The flow path of the distillate is focused to drop into a $0.05-\mathrm{mL}$ "hammock" that is positioned directly below the flow path. The distillate from the condenser drops into this volume before it falls into the receiver. A crimp cap fixture is incorporated as a side arm of the adapter. This allows a replaceable crimp cap with a silicone or Teflon septum (of the type used for chromatographic automatic sampler vials) to be positioned in line with the hammock. The distance from the crimp cap to the base of the hammock is suited to the needle length of typical gas chromatographic syringes.

To sample the distillate, one simply positions the chromatographic syringe equipped with a blunt tipped needle in the well of the hammock. It is a simple matter to withdraw samples as a function of distillate volume when a calibrated receiver is used for collection. The sample can then be directly injected into the gas chromatograph, or added to a weighed vial containing an appropriate solvent.

When the sample drops from the sampling transfer adapter, it flows into the calibrated receiver to allow a volume measurement. As mentioned 
earlier, the simple graduated cylinder that is often used for the volume measurement (and that is illustrated in ASTM-86) is not optimal because of the relatively large uncertainty, and the fact that distillate splashes into the cylinder, causing difficulty in determining the volume as it changes through the course of the measurement. The use of drop deflectors can help prevent splashing but they can cause a higher uncertainty in the volume measurement because of hold-up on the walls of the receiver. Automated optical level followers make the volume measurement easier, but they can also be a source of uncertainty, and they make it impossible to sample the distillate because of their placement near the receiver. To improve the precision of the volume measurement, the calibrated receiver that is shown in Fig. 3 was developed. Constructed of glass, this receiver consists of a central volume that gradually decreases in diameter at the base, and connects to a small-diameter side arm sight glass that is calibrated. The side arm stabilizes the fluid level for a precise volume measurement as the distillation proceeds. The large inner volume and the sight glass are enclosed in a water jacket that contains a thermometer and a magnetic stir bar for circulation. The tube of the sight glass is glasswelded to the inside wall of the water jacket, to eliminate parallax in reading the volume. The water jacket allows the receiver to be maintained at a suitable temperature, and the stirrer ensures acceptable temperature uniformity. The availability of these two sizes allows measurement of fluids with varying surface tensions. The side arm sight glass allows a volume measurement with an uncertainty of $0.05 \mathrm{~mL}$. The sight glass is calibrated with the volumes of interest to the distillation curve $(10-200 \mathrm{~mL}$, in $10 \mathrm{~mL}$ increments) with a volumetric pipette.

The volume measurement in the side arm is unaffected by splashing in the collection volume, so the distillate is allowed to drop directly into the center of the collection volume space. This minimizes hold-up on the walls of the vessel. Splashing causes some hold-up, but this is much less than when a deflector is used. The base of the receiver is equipped with two bulls-eye type levels $90^{\circ}$ apart to ensure that the liquid level in the large volume and the side arm are co-linear.

To perform a measurement, $200 \mathrm{~mL}$ of the fluid to be measured is placed in the round bottom flask (typically with $100 \mathrm{~mL}$ pipettes), and the thermocouples are placed in the appropriate positions. This fluid volume has been chosen for convenience; any volume suitable for the measurement can be used. In the early work with this apparatus, heat was applied to the flask either under manual or PID control. Note that the temperature measurement made directly in the fluid is unaffected by heating rate, since it represents a true thermodynamic state point. The temperature measured in the head is very dependent on heating rate, however, and it is necessary 
to address this rate problem to maintain continuity with historical data. For this reason, it is best to maintain a constant mass-transfer rate during the distillation. In commercial devices, this behavior is approximated with a PID controller, with a response fed back into the heater controller.

In our previous work, we presented measurements on simple binary mixtures of $n$-decane $+n$-tetradecane, as test fluids for the new approach [4]. For different starting mole fractions of that binary, we measured distillation curves that exhibited the familiar sigmoidal shape, an example of which (for a $50 / 50 \mathrm{~mol} \%$ mixture) is shown in Fig. 4. The curve presented here represents the temperature (corrected for ambient atmospheric pressure) measured directly in the fluid, $T_{\mathrm{k}}$ (note that the sudden jump in temperature indicating lift-out, as discussed earlier, is clearly visible at the highest volume fraction). The more common approach is to measure the temperature in the distillation head, represented as $T_{\mathrm{h}}$. A common difficulty with this temperature measurement location is that even slight variations in mass-flow rate through the head will cause aberrations in

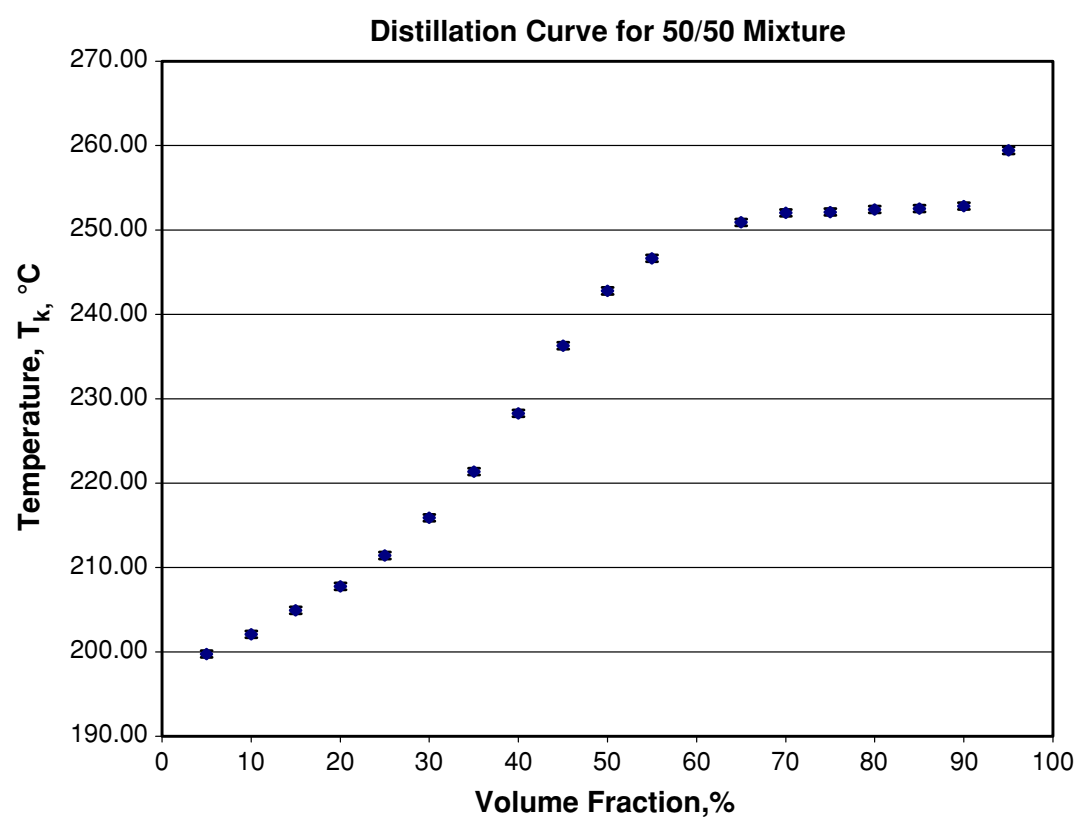

Fig. 4. Representative distillation curve for a $50 / 50 \mathrm{~mol} \%$ mixture of $n$-decane $+n$-tetradecane, measured with $\mathrm{T} 1$ inside the fluid. Note that the second inflection at a volume fraction of approximately $97 \%$ is the lift-out of T1, as explained in the text. It is not part of the distillation curve proper, but it is an indicator of the end of a measurement. Error bars represent a combined uncertainty of two standard deviations. 
$T_{\mathrm{h}}$. Such aberrations are sometimes called hesitation [2]. This can be seen in Fig. 5, in which a $T_{\mathrm{h}}$ curve is presented along with a $T_{\mathrm{k}}$ curve for the same $50 / 50 \mathrm{~mol} \%$ starting mixture. This measurement differs from that shown in Fig. 4 in that the heating rate was held constant throughout the measurement, thus causing a changing mass-flow rate through the head in response to changing fluid composition. The behavior of the $T_{\mathrm{k}}$ curve is unaffected by heating rate; it is controlled only by the fluid behavior. Clearly, the $T_{\mathrm{h}}$ curve does not represent the actual fluid behavior, and it is unreliable and not repeatable.

In this paper, we present an approach in which a model predictive temperature controller heats the distillation flask enclosure such that the temperature of the enclosure leads the temperature of the fluid by a pre-selected value. The temperature profile applied to the enclosure is calculated either with an equation-of-state [7] or it is determined from a previous distillation curve measurement of the fluid [6]. Instead of testing with a prepared binary mixture, in these studies we used a standard 91 research octane number (RON) gasoline and oxygenated mixtures thereof.

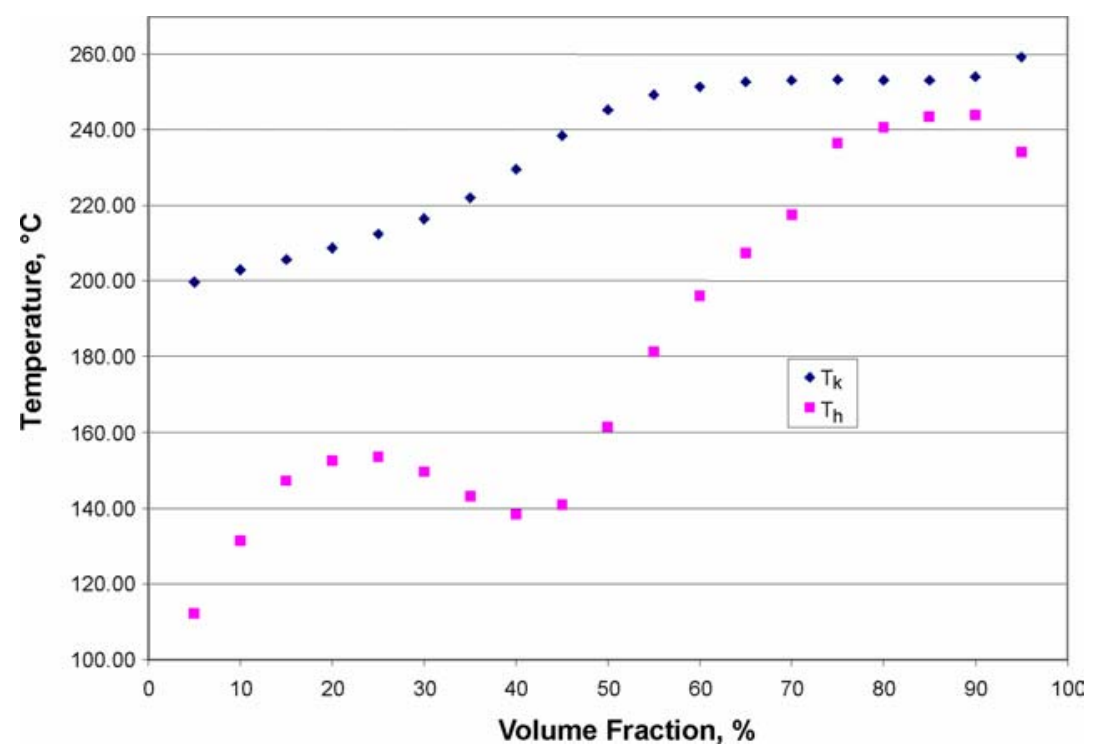

Fig. 5. Distillation curve for a $50 / 50 \mathrm{~mol} \%$ mixture of $n$-decane $+n$-tetradecane, measured directly in the fluid ( $T_{\mathrm{k}}$ measured in the kettle) and in the distillation head $\left(T_{\mathrm{h}}\right.$, measured in the head), when a constant heating rate is applied to the distillation flask. Diverging points at a volume fraction of $95 \%$ signify the end of the measurement. 


\section{EXPERIMENTAL}

The distillation curve measurements used for testing the model predictive temperature controller were performed on a 91 antiknock index (AI) gasoline obtained from a commercial supplier, and used without treatment. This kind of fuel sample represents a test fluid that is much more akin to samples that must be measured industrially on a routine basis [8-11]. We maintained the sample in a sealed container at $7^{\circ} \mathrm{C}$, to ensure that no compositional changes would occur during the course of our measurements. In addition, we prepared mixtures of this gasoline with methanol, to simulate oxygenates. The mixtures were prepared volumetrically at ambient temperature with graduated cylinders.

In Fig. 6, we present the measured distillation curves for the gasoline, and for mixtures of this base gasoline with $10 \%$ and $15 \%$ methanol ( $\mathrm{vol} / \mathrm{vol}$ ). These curves are presented in $T_{\mathrm{k}}$, and while they are thermodynamically sound, they are not directly comparable to historical data presented in $T_{\mathrm{h}}$. The base gasoline curve shows a subtle sigmoidal shape

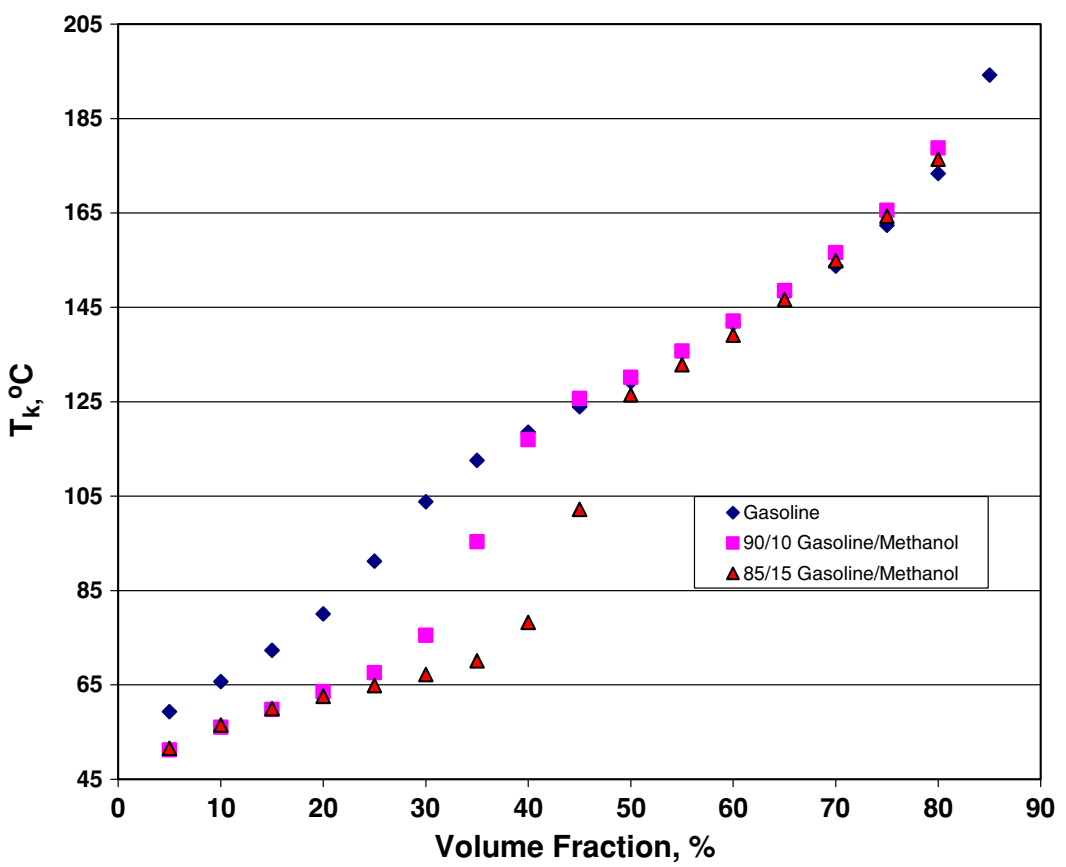

Fig. 6. Measured distillation curves of a $91 \mathrm{AI}$ gasoline, and mixtures of this base gasoline with $10 \%$ and $15 \%$ methanol ( $\mathrm{vol} / \mathrm{vol})$. This figure illustrates the multiple inflections that are common in the distillation curves of oxygenated gasolines. 
that is typical for a complex fluid. As the fluid becomes more and more polydisperse in terms of components, the classical sigmoid shape (for example, of the binary shown in Fig. 4) flattens and becomes more linear [6]. The addition of methanol causes two obvious effects. First, the appearance of additional inflections in the lower volume fraction range of the curve can be seen. This is a well-known aspect of oxygenated gasolines [12]. Second, also in the lower volume fraction range, we see a decrease in temperature, indicating that the "lighter ends" of the gasoline have become more volatile. In this range, the distillation temperatures of the cuts are between $8^{\circ} \mathrm{C}$ and $10^{\circ} \mathrm{C}$ below that of the base gasoline. The later volume fractions are unaffected by the addition of methanol.

Starting with either a previously measured distillation curve or a calculated distillation curve (from an equation-of-state [7]), one can improve the measurement of distillation curves and reliably measure both $T_{\mathrm{k}}$ and $T_{\mathrm{h}}$. This is done by imposing a heating profile on the distillation flask enclosure such that the enclosure temperature leads the fluid temperature by some pre-selected temperature, for example $20^{\circ} \mathrm{C}$. In this way, one can achieve a constant mass-flow rate through the distillation head, and a measured head temperature that will be unaffected by rate aberrations so common in distillation curve measurement.

Heating profiles are programmed on a commercial temperature controller with guidance from the distillation curve shape (measured or calculated) of the fluid under study. A arbitrary number of "steps" can be programmed into the controller in order to closely follow the trend of the distillation curve. The only major difference between the distillation curve itself and the enclosure heating profile is that the programmed heating profile is set to be higher in temperature, and this difference is maintained approximately constant (within $2^{\circ} \mathrm{C}$ ) throughout the profile. The number of "steps" in the program is determined by the number of segments in the distillation curve that can be approximated as linear trends. For each major change in slope of the curve, a separate step is used to match its trend. Further, dependent upon the steepness of the slope of the linear segments, a specific energy input is specified for that particular "step." Steeper slopes are generally programmed with higher energy inputs. Thus, this furnishes the two major parameters of control. The first is the initial and final temperatures of a particular step, and the second is the energy input available during that step. An additional control parameter that is available is the constant temperature "soak." This is a waiting period of constant temperature that is applied to the enclosure by the controller. These waiting periods are used to ensure that the enclosure does not overrun the fluid temperature at the end of a step. They are also useful when a fluid has boiled to a single component at the end of a distillation curve measurement. 
The heating profile that is programmed must be tested for a given fluid by monitoring the head temperature of the distillation curve apparatus for any fluctuations or evidence of hesitation. If fluctuation or hesitation is noted, the heating profile must be revised by changing the rate of "steps" or by altering the temperatures of the linear segments of the programs. There is always a lag between the temperature of the temperature controller and that of the temperature of the fluid, and the desire is to maintain this difference as a constant throughout the measurement.

Once a heating profile is programmed and tested, it can be used for many fluids that are similar in behavior. Thus, simple binaries and gasolines have very similar behavior, and thus the same program may be used for such fluids. Moreover, changes in the heating profile are easy to apply. As examples of heating profiles that we have found successful, in Fig. 7a we present a profile for the binary of $n$-decane $+n$-tetradecane, and in Fig. $7 \mathrm{~b}$, the profile for a gasoline containing $15 \%$ methanol (vol $/ \mathrm{vol})$.

\section{RESULTS AND DISCUSSION}

The heating profiles discussed above have been used to measure distillation curves of a number of complex fluids, among them, gasolines and oxygenated gasolines, aviation turbine fuels, and rocket propellants. In Fig. 8a, we present the distillation curve for $91 \mathrm{AI}$ gasoline. In this figure, $T_{\mathrm{k}}$ is the temperature measured directly in the fluid, and $T_{\mathrm{h}}$ is that measured in the head (the usual location for the temperature measurement). Clearly, the readings of $T_{\mathrm{k}}$ and $T_{\mathrm{h}}$ track very reliably. There is an offset in the two readings because there is a short delay in the fluid leaving the distillation flask and its arrival in the head. There is no evidence of hesitation or mass-flow-related aberrations. The divergence that is seen at a volume fraction of $90 \%$ is due to lift-out of the thermocouple from the fluid (the thermocouple measuring $T_{\mathrm{k}}$ ). This point in the $T_{\mathrm{k}}$ plot is not part of the distillation curve proper, merely an indicator of the end of the measurement.

A more challenging example is gasoline with $15 \%$ methanol. As seen in Fig. 6, this fluid has a complex distillation curve with multiple inflection points. It is particularly important in cases such as these that the massflow rate through the distillation head be constant. We measured this fluid with a heating profile that is somewhat more complicated, as shown in Fig. 7b. Despite the increased complexity, the curves in $T_{\mathrm{k}}$ and $T_{\mathrm{h}}$ on Fig. $8 \mathrm{~b}$ track one another very well, even in the inflection areas.

The mass-flow rate of fluid through the distillation head is easily measured at the receiver. One can measure the time rate of change of mass (or volume, if a density is available) in the receiver, or simply measure the 

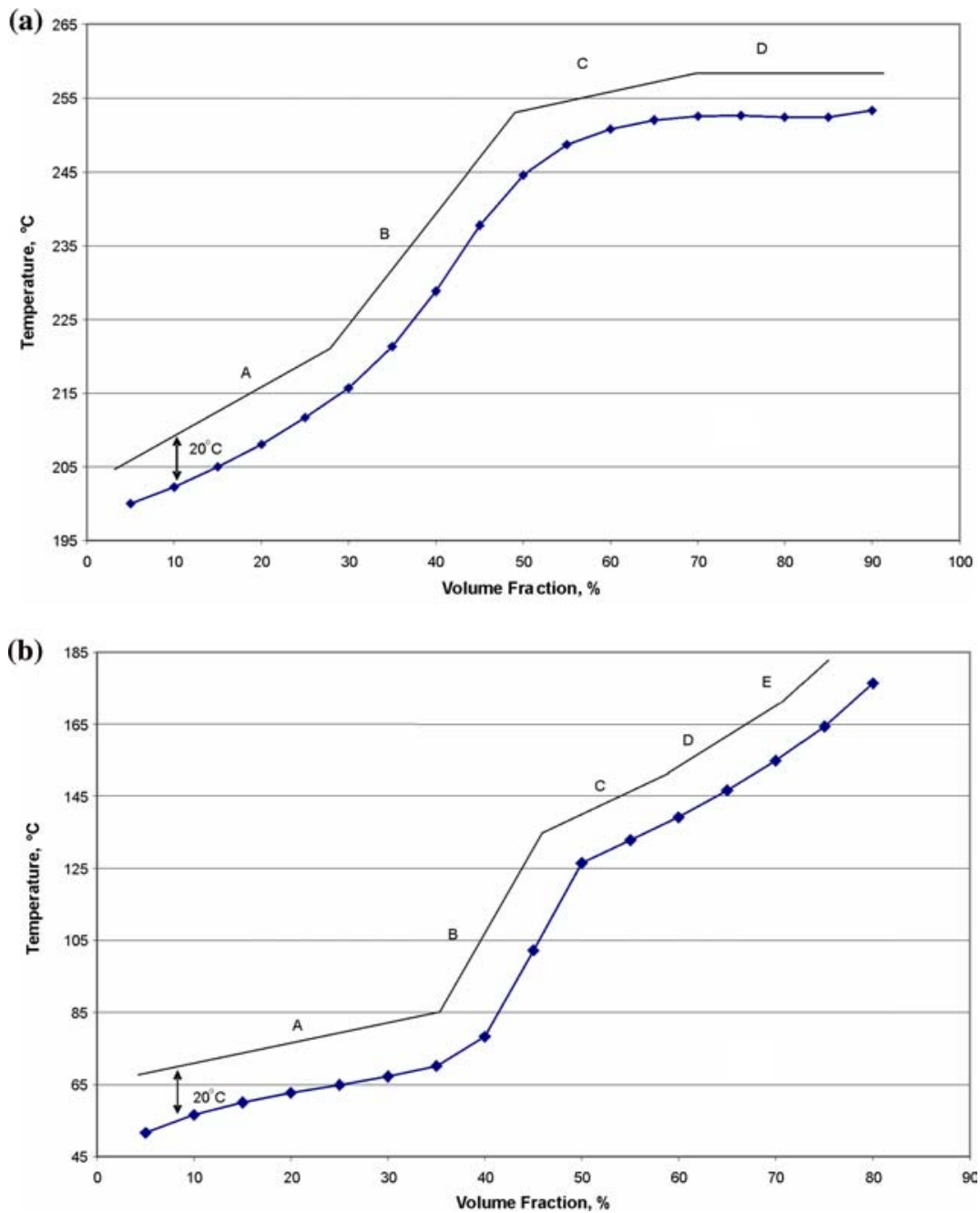

Fig. 7. (a) Plot showing the distillation curve for $n$-decane $+n$-tetradecane, and the heating profile programmed to maintain an approximately constant mass-flow rate through the distillation head. (b) Plot showing the distillation curve for 91 AI gasoline with $15 \%$ methanol $(\mathrm{vol} / \mathrm{vol})$, and the heating profile programmed to maintain a constant mass-flow rate through the distillation head.

time required for a specified number of droplets to fall into the receiver. We have found that using the controller produces an average uncertainty in flow rate of $2.1 \%$. Thus, during a typical measurement for gasoline, the mass flow rate is maintained at $1.85 \mathrm{~g} \cdot \mathrm{min}^{-1}$, with an uncertainty of 0.05 . 

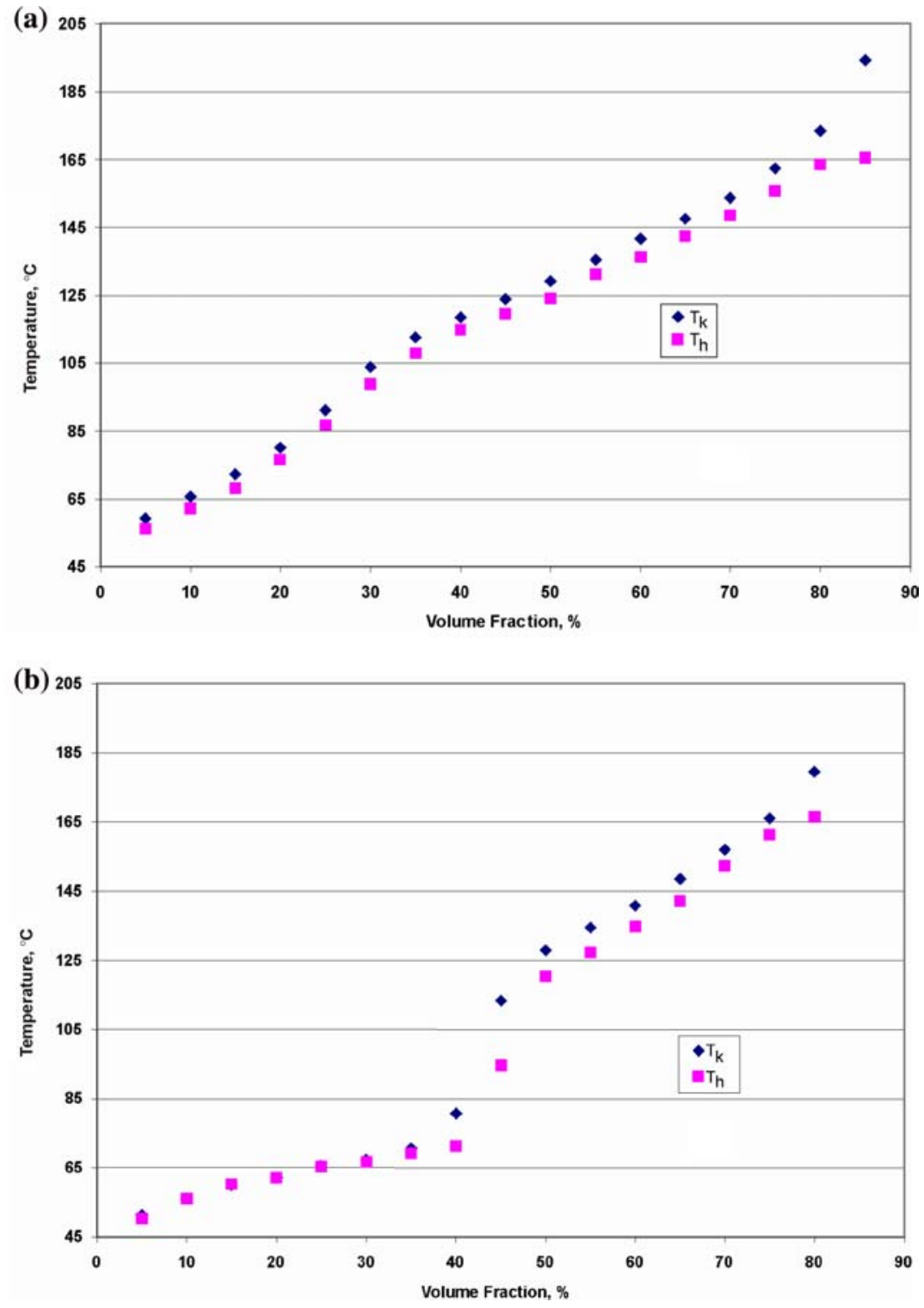

Fig. 8. (a) Distillation curves of $91 \mathrm{AI}$ gasoline, presented in $T_{\mathrm{k}}$ and $T_{\mathrm{h}}$, measured with the model predictive temperature controller programmed with a heating profile designed to achieve a constant mass-flow rate through the distillation head. (b) Distillation curves of 91 AI gasoline with $15 \%$ methanol ( $\mathrm{vol} / \mathrm{vol}$ ), presented in $T_{\mathrm{k}}$ and $T_{\mathrm{h}}$, measured with the model predictive temperature controller programmed with a heating profile (shown in Fig. 7b) designed to achieve a constant mass-flow rate through the distillation head. 


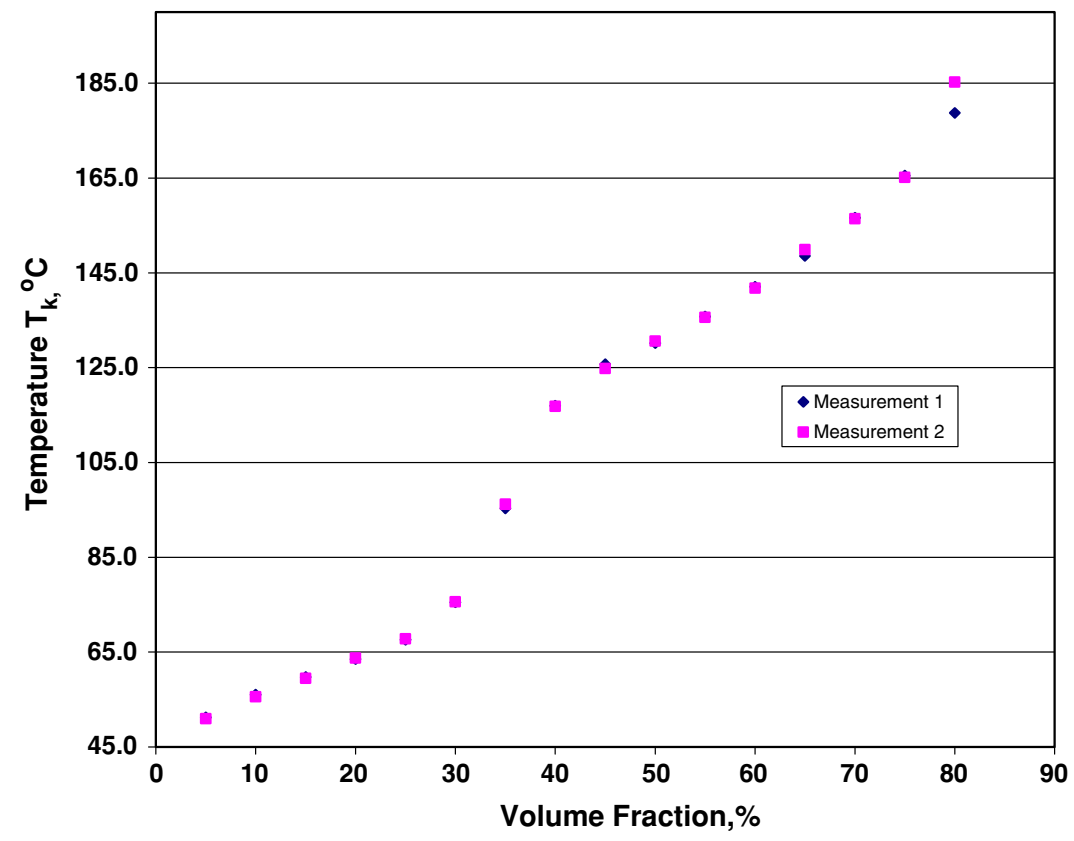

Fig. 9. Two distillation curves for 91 AI gasoline with $10 \%$ methanol, illustrating the repeatability of complex distillation curves measured with the model predictive temperature controller.

An additional benefit that results from the new temperature control approach is a significant decrease in curve uncertainty. In the earlier paper discussing our initial improvements, we reported that the combined expanded uncertainty $(k=2)$ of distillation curve temperatures had been decreased from the usual $5^{\circ} \mathrm{C}$ to $10^{\circ} \mathrm{C}$ to approximately $0.2^{\circ} \mathrm{C}$. With the model predictive temperature controller, we have further decreased the uncertainty that is obtainable to a level less than approximately $0.1^{\circ} \mathrm{C}$. Moreover, this uncertainty is obtainable for both the $T_{\mathrm{k}}$ and $T_{\mathrm{h}}$ curves. We illustrate this repeatability in Fig. 9, in which two curves for the base gasoline with $10 \%$ methanol are shown.

\section{CONCLUSIONS}

We have presented an approach to the measurement of distillation curves that represents a significant advancement over previous techniques. Our initial improvements included the addition of a composition channel of data to the distillation data, and temperature and volume measurements 
of lower uncertainty. Moreover, our approach to measurement of the fluid temperature directly $\left(T_{\mathrm{k}}\right)$ provided data that were amenable to thermodynamic modeling, since actual state-point measurements were made. The improvement we have reported in this paper uses a model predictive temperature controller to control the heating profile and obtain constant mass-flow rate through the distillation head. This improvement further refines our approach in that we can now directly relate our state-point measurements to the historical data that have been developed over the last century (presented with $T_{\mathrm{h}}$ ). While we do not advocate the presentation of distillation data in terms of $T_{\mathrm{h}}$, the measurement of both $T_{\mathrm{k}}$ and $T_{\mathrm{h}}$ is an advantage. We have demonstrated that the heating profiles provide distillation curves that are free from hesitation or flow-related aberrations.

\section{ACKNOWLEDGMENT}

BLS gratefully acknowledges a Professional Research Experience Program (PREP) undergraduate fellowship at NIST.

\section{REFERENCES}

1. W. L. Leffler, Petroleum Refining in Nontechnical Language (PennWell, Tulsa, Oklahoma, 2000), Chaps. 1 and 2.

2. Standard Test Method for Distillation of Petroleum Products at Atmospheric Pressure, ASTM Standard D 86-04b, Book of Standards Vol. 05.01. 2004 (American Society for Testing and Materials, West Conshohocken, Pennsylvania, 2004).

3. N. Babinsky, Koehler Instrument Company, Inc., personal communication (2005).

4. T. J. Bruno, Ind. Eng. Chem. Res. 45:4371 (2006).

5. T. J. Bruno, Sep. Sci. Technol. 41:309 (2006).

6. T. J. Bruno and B. L. Smith, Ind. Eng. Chem. Res. 45:4381 (2006)

7. E. W. Lemmon, M. O. McLinden, and M. L. Huber, REFPROP, Reference Fluid Thermodynamic and Transport Properties, NIST Standard Reference Database 23 (National Institute of Standards and Technology, Gaithersburg, Maryland, 2005).

8. V. E. Emel'yanov, V. P. Grebenshchikov, V. F. Golosova, and G. N. Baranova, Khimiya $i$ Tekh. Top. i Masel 11:22 (1982).

9. A literature review based assessment on the impacts of a $20 \%$ ethanol gasoline fuel blend on the Australian vehicle fleet, in Report to Environment Australia (Orbital Engine Company, Balcatta, Western Australia, 2003).

10. S. H. Choi, G. B. Kim, Y. J. Chang, and C. H. Jeon, Int. J. Automotive Technol. 5:311 (2004).

11. B. Visser, Gen. Aviations News 1 (April 2004), p. 1.

12. A literature review based assessment on the impacts of a $10 \%$ and $20 \%$ ethanol gasoline fuel blend on non-automotive engines, in Report to Environment Australia (Orbital Engine Company, Balcatta, Western Australia, 2002). 